\title{
Orbital Compartment Syndrome with Traumatic Optic Neuropathy: A Case Report and Literature Review
}

\author{
Mohamed Elshikh*, Irfan Masood, Woolridge N and Heymann J \\ Department of Diagnostic Radiology, The University of Texas Medical Branch, Galveston, Texas \\ *Corresponding author: Mohamed Elshikh, Department of Diagnostic Radiology, Galveston, Texas
}

\begin{tabular}{|c|c|}
\hline ARTICLE INFO & ABSTRACT \\
\hline Received: 仹 October 01, 2019 & Abbreviations: VA: Visual Acuity, IOP: Intraocular Pressure, ON: Optic Nerve, Traumatic \\
\hline Published: 閥October 09, 2019 & $\begin{array}{l}\text { Optic Nerve Neuropthy, OCS: Orbital Compartment Syndrome, DTON: Direct Traumatic } \\
\text { Optic Neuropathy }\end{array}$ \\
\hline $\begin{array}{l}\text { Citation: Mohamed Elshikh, Irfan } \\
\text { Masood, Woolridge N, Heymann J. Orbital } \\
\text { Compartment Syndrome with Traumatic } \\
\text { Optic Neuropathy: A Case Report and } \\
\text { Literature Review. Biomed J Sci \& Tech } \\
\text { Res 21(5)-2019. BJSTR. MS.ID.003660. }\end{array}$ & \\
\hline
\end{tabular}

\section{Introduction}

\section{Clinical History}

49-years-old male presented to the ED with history of right orbital trauma. Physical examination showed right periorbital swelling, multiple periorbital lacerations, restricted right eye extraocular movements, and decreased Visual Acuity (VA). Intraocular Pressure (IOP) was elevated at $52 \mathrm{mmHg}$. Upon presentation, an emergent bedside lateral canthotomy and inferior cantholysis were performed. Postoperatively, the IOP has decreased to $31 \mathrm{mmHg}$. 24 hours after the procedure, the IOP and VA were $26 \mathrm{mmHg}$ and 20/100, respectively. However, patient reported complete right eye visual loss. IOP was persistently elevated. Anterior orbitotomy was performed for further decompression.

\section{Imaging Findings}

The initial CT after the lateral canthotomy and inferior cantholysis showed a displaced fracture of the right inferior orbital floor with herniation of the inferior rectus muscle in the right maxillary sinus, comminuted fracture of the lamina papyracea (Figures 1a-1c) Marked proptosis and retrobulbar fat stranding and hematoma were noted (Figure $1 \mathrm{~d}$ ). The posterior globe angle was 115 degrees (Figure 1b) Consistent with severe orbital compartment syndrome. With worsening vision, orbital MRI was obtained 24 hours after admission and showed decreased posterior globe angle to 99 degrees (Figures 2a \& 2b) with more evident posterior globe tenting. The Optic Nerve (ON) was hyperintense on DWI and hypointense on ADC map indicating Traumatic Optic Nerve Neuropthy (TON) (Figure 2c) 

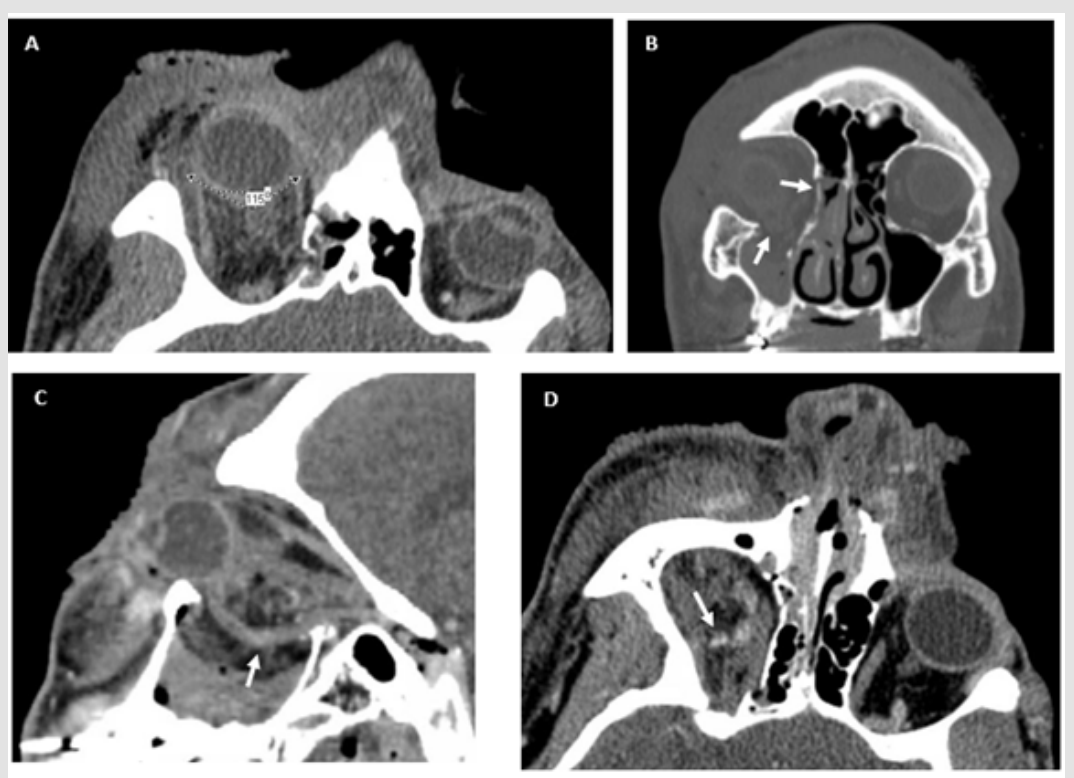

Figure 1: Non-contrast CT scan of the orbit (a) axial view shows marked right eye proptosis, right facial soft tissue swelling, and severe globe tenting with a posterior globe angle of 115 degrees. (b) Coronal view demonstrates inferior and medial orbital walls fractures (arrow) with right maxillary hemosinus. (c) Note herniation of the inferior rectus muscle and intraorbital fat (arrows) through the inferior wall fracture on the saggital view. A retrobulbar hematoma (arrow) is seen on (d).
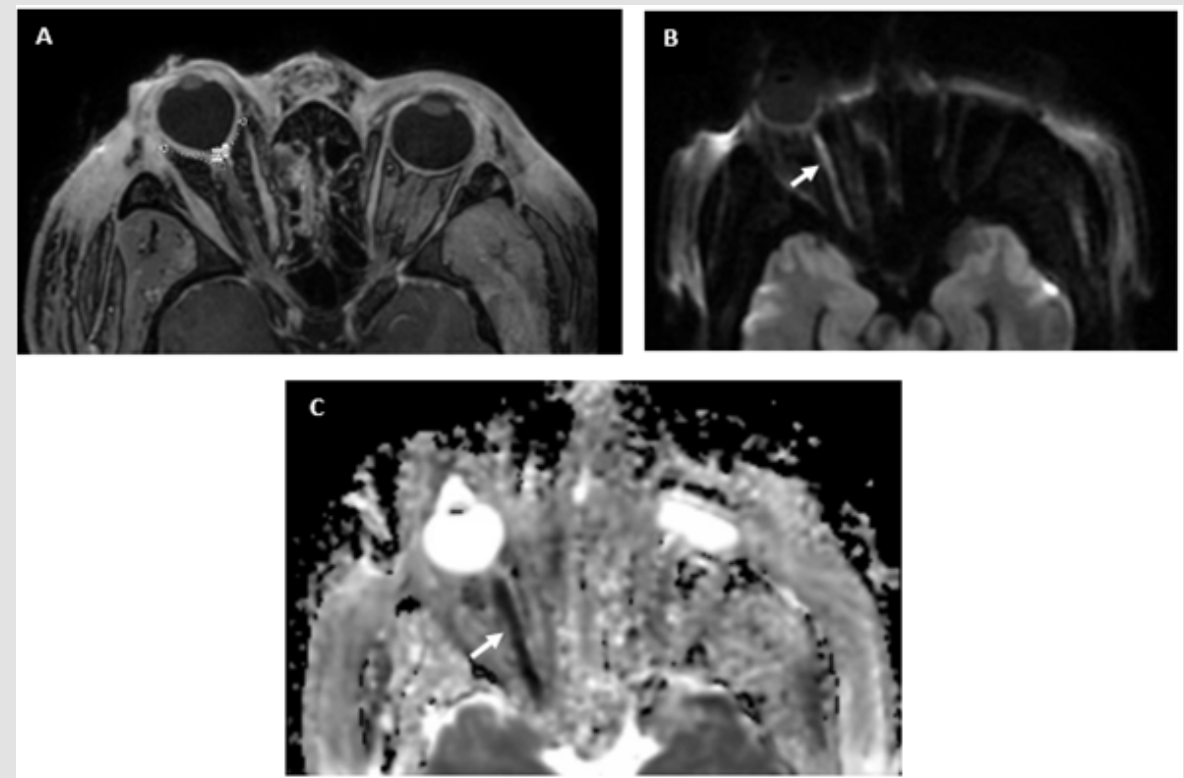

Figure 2: Contrast enhanced MRI of the orbit. (a) Axial postcontrast T1-WI MRI done 24 hours after admission shows worsening of right globe tenting with posterior globe angle of 99 degrees decreasing from 115 degres on admission (Fig. 1a).

(b) Axial diffusion weighted MRI of the orbit demenostrates high right optic nerve signal (arrrows) that is low on ADC map

(c), consistend with TON.

\section{Discussion}

Orbital Compartment Syndrome (OCS) occurs when the orbital pressure acutely rises causing perfusional impairment of the intraorbital structures including the Optic Nerve (ON) [1,2]. OCS may occur following facial trauma, as in our case. Visual loss is the most devastating complication of OCS. Visual loss occurs as a result of or vascular or neurological compromise of the central retinal artery or optic nerve. OCS is a clinical diagnosis that requires promptinterventions. Clinically, patient may present with proptosis, periorbital swelling, double vision, and/or TON symptoms. Clinical examination usually shows increased Intraocular Pressure (IOP), restricted ocular movement, and protptosis [2]. Management of OCS includes urgent decompression with lateral canthotomy and inferior cantholysis $[3,4]$. Proptosis and increased IOP may lead to stretch and ischemic Injury of The Optic Nerve (TON), as seen in our patient. 
ON is anatomically composed of four segments; Intraocular, intraorbital, intracanalicular, and intracranial. TON describes any ON injury due to trauma. The intracanalicular segment is more prone to TON followed by the intraorbital segment, especially at the orbital apex. TON is a rare entity with an incidence of $0.7-2.5 \%$ in patients with significant head trauma with higher prevalence in males [3-5]. TON can be due to direct or indirect injury. Indirect Traumatic Optic Neuropathy (ITON) occurs when the shock forces are transmitted to the optic canal from a remote location. While, Direct Traumatic Optic Neuropathy (DTON) occurs due ON transection, avulsion, or compression as seen in our case [3-5]. ITON is more common than DTON [4]. Clinically, patients with TON may have variable visual loss, visual field defects, relative afferent papillary defect, or color vision impairment [3-6].

Imagining should not delay therapeutic interventions for OCS or TON. Imaging plays an important role in confirming the diagnosis and revealing the underlying pathology like hematoma (Figure 1d) and cellulitis. The salient imaging feature for acutely rising retrobulbar pressure is globe tenting. Globe tenting is a conical configuration of the globe posteriorly where the optic nerve inserts (Figure 1a) [7,8]. Globe tenting can be quantitatively assessed by measuring the posterior globe angle. The posterior globe angle is the angle between two tangents extending from the optic nerve insertion to the sclera medially and laterally (Figure 1a).

Normally, this angle should measure more than 130 degrees. An angle less than 130 degrees is pathological. According to the posterior globe angle, the tenting is classified as mild (posterior globe angle between 120 - 130 degrees) and severe (posterior globe angle $<120$ degree) as seen in our case (initial angle was 115, (Figure 1a) and decreased to 99 degrees, (Figure 2a). With severe tenting, emergent intervention should be pursued to preserve vision. Patients with mild globe tenting usually have a better outcome and a higher chance of complete recovery [7]. The globe tenting occurs due to acute or subacute proptosis associated with suddenly increasing retrobulbar pressure. The acute axial displacement leads to $\mathrm{ON}$ stretch, contusion and/or ischemia which may lead to ITON $[7,8]$. Radiographically, MRI plays an essential role in diagnosing TON, especially in altered patients due to insufficient clinical examination. DWI and ADC sequences are vital for TON. High signal intensity on DWI with corresponding low signal on ADC maps is $28 \%$ sensitive and $100 \%$ specific for TON (Figures 2 b \& 2c). Quantitative comparison of ADC values between the affected and non-affected $\mathrm{ON}$ is a helpful tool for diagnosing TON. The injured $\mathrm{ON}$ have a lower $\mathrm{ADC}$ value than the intact $\mathrm{ON}$. This is significant in the early post injury period ( $\leq 7$ days) [9].

\section{References}

1. Cheng ACO (2017) Orbital Compartment syndromes. Emergencies of the Orbit and Adnexa.

2. Lima V, Burt B, Leibovitch I, Prabhakaran V, Goldberg RA, et al. (2009) Orbital Compartment Syndrome: The Ophthalmic Surgical Emergency. Survey of Ophthalmology 54(4): 441-449.

3. Kumaran AM, Sundar G (2015) Traumatic Optic Neuropathy: A Review. Craniomaxillofac Trauma Reconstruction 8(1): 31-41.

4. Singman EL (2016) Indirect traumatic optic neuropathy. Military Medical Research 3: 2 .

5. Yu Wai Man P, Griffiths PG (2013) Surgery for traumatic optic neuropathy Cochrane Database of Systematic Reviews 19(4): CD005024.

6. Levin LA, Beck RW, Joseph MP, Seiff S, Kraker R (1999) The international Optic Nerve Trauma Study. Ophthalmology 106: 1268-1277.

7. Dalley RW, Robertson WD, Rootman J (1987) Globe Tentin: A Sign of Increased Orbital Tension. AJNR 10: 181-186.

8. Charles JH, Douglas JQ (1996) Globe Tenting as a Result of Head Trauma. AJNR 18: 980-982.

9. Bodanapally UK, Dreizin D, Katzman L, Reddy RP (2015) Hyperintense Optic Nerve due to Diffusion Restriction: Diffusion-Weighted Imaging in Traumatic Optic Neuropathy. AJNR 36: 1536-1541.

\section{ISSN: 2574-1241}

DOI: 10.26717/BJSTR.2019.21.003660

Mohamed Elshikh. Biomed J Sci \& Tech Res

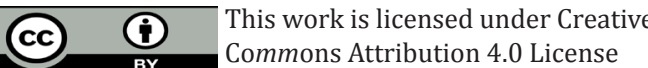

Submission Link: https://biomedres.us/submit-manuscript.php

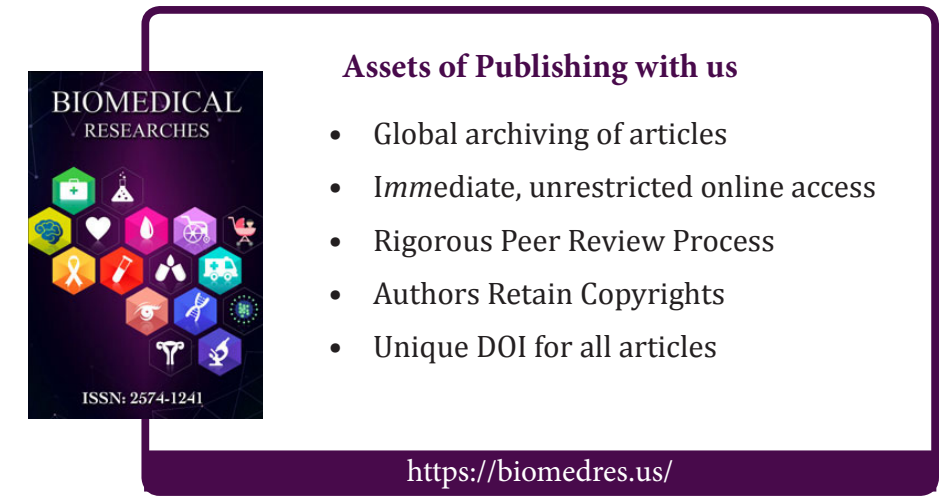

